\section{Algunas reflexiones a propósito de la publicación del décimo volumen de la revista Anuario Latinoamericano - Ciencias Políticas y Relaciones Internacionales}

\author{
Some Thoughts on the Occasion of the Publication \\ of the Tenth Volume of the Journal Anuario Latinoamericano \\ - Ciencias Políticas y Relaciones Internacionales
}

Anuario Latinoamericano Ciencias Políticas

y Relaciones Internacionales vol. 10, 2020

pp. 229-238

DOI: 10.17951/al.2020.10.229-238
La publicación del décimo volumen de la revista Anuario Latinoamericano Ciencias Políticas y Relaciones Internacionales me ha motivado a compartir con nuestros lectores algunas reflexiones sobre el trabajo científico y editorial que se viene realizando de forma constante y sistemática desde hace siete años. Después de publicar diez volúmenes de la revista ya podemos resumir la experiencia adquirida y reflexionar sobre el desarrollo del Anuario Latinoamericano.

Desde la creación de la revista en 2014, nos hemos atenido a tres principios fundamentales: incrementar constantemente el nivel de calidad, aumentar su visibilidad y desarrollar el impacto en el entorno académico de los investigadores latinoamericanistas en Polonia y en el extranjero (Krzywicka, 2018, pp. 158-167; Krzywicka, 2018a, pp. 299-302). A esos tres principios fundamentales de la filosofía y estrategia de la revista Anuario Latinoamericano, conviene añadir otros valores, que no solo los complementan, pero también los condicionan: la coherencia, la transparencia, la diversidad, la pluralidad, el intercambio y la integración, la internacionalización y, last but not least, la regularidad y la accesibilidad. Al principio, la revista fue pensada -tal como indica su nombre- como una publicación anual. Sin embargo, al aumentar su visibilidad y el reconocimiento en el entorno de los estudiosos latinoamericanistas, en 2019 decidimos transformarla en una publicación semestral y esperamos que a partir de 2021 se publicará con frecuencia trimestral.

Hay varios factores que deciden sobre la calidad de una revista científica. No se trata solo de la selección cuidadosa de autores y temas. También son importantes tales elementos como la aplicación del proceso de la revisión de doble ciego, el uso de un programa antiplagio o una estructura bien pensada para organizar el contenido de cada volumen. En nuestro caso, hemos decidido basar la estructura sobre tres secciones: una sección monográfica de "Dossier", gestionada por coordinadores científicos invitados para cada volumen, 
la sección "Artículos y ensayos" y la sección "Reseñas e informes". El tema seleccionado en el "Dossier" puede ser inspirado por alguna cuestión actualmente discutida en la comunidad científica de latinoamericanistas o puede ser propuesto a potenciales autores para iniciar un debate sobre unas cuestiones originales e importantes y además menos investigadas. El segundo apartado de la revista, titulado "Artículos y Ensayos", cumple una función complementaria muy importante, ya que permite a todos los autores publicar los resultados de su investigación, independientemente del tema principal previsto para el "Dossier" del volumen. La tercera sección mencionada, "Reseñas e informes", fue inaugurada en el segundo número del Anuario Latinoamericano en 2015 con el fin de, primero, enriquecer el debate científico con reseñas de novedades editoriales y, segundo, difundir entre la comunidad de investigadores latinoamericanistas las informaciones sobre las actividades que sirven para la divulgación del trabajo científico, tales como simposios, conferencias y congresos celebrados en Polonia y en otros países del mundo.

De acuerdo con los principios mencionados, el papel de la revista Anuario Latinoamericano consiste en integrar el entorno de los politólogos latinoamericanistas y profundizar la colaboración científica, permitiendo el intercambio de opiniones y resultados de investigación entre científicos de diferentes centros académicos nacionales y extranjeros, contribuyendo así a la internacionalización de la investigación y al desarrollo de la cooperación internacional científica. Es motivo de gran satisfacción haber podido colaborar en la preparación de los diez volúmenes de nuestra revista con 145 autores y 96 evaluadores provenientes de 28 países. Entre ellos se encuentran representantes de Argentina, Belice, Bolivia, Brasil, Chile, Colombia, Guatemala, México, Paraguay, Perú, Uruguay, Venezuela, Alemania, China, Estados Unidos, España, Francia, Reino Unido, Grecia, Israel, Lituania, Polonia, Portugal, República Checa, Rusia, Serbia, Suiza, Suecia. En total, hemos publicado 124 artículos, 22 reseñas de libros y 16 informes sobre eventos científicos en el campo de los estudios latinoamericanos, que tuvieron lugar en Polonia y en el extranjero.

La revista aparece en la versión impresa, pero también es disponible en línea, partiendo de los principios de la política de acceso abierto. Esto significa que hay acceso libre, gratuito y rápido al contenido de la versión electrónica de cada de los diez volúmenes publicados del Anuario Latinoamericano. El usuario tiene acceso a todos los materiales sin restricciones financieras, jurídicas o técnicas, si bien debe respetar los derechos del autor.

Ahora pasaremos a una breve descripción del contenido de los diez volúmenes publicados desde la fundación de la revista en 2014. Aparte de ser editora en jefe y directora de la revista, también cumplo el papel de la coordinadora científica, pero en la preparación de casi cada volumen ha participado un coordinador invitado, especialista en el tema del "Dossier" particular.

El primer volumen, titulado "América Latina: cambios a nivel regional y en su inserción internacional”, fue preparado bajo la coordinación científica de Martha Ardila de la Universidad Externado de Colombia y la mía. El tema del 
volumen surgió en el contexto del debate científico que tuvo lugar en el 54 Congreso Internacional de Americanistas (ICA) en Viena (2012), como parte de un panel organizado y moderado por las coordinadoras del "Dossier" del volumen 1/2014 del Anuario Latinoamericano (Ardila y Krzywicka, 2014, pp. 13-15).

El tema del segundo volumen (2/2015), preparado bajo mi coordinación científica, fueron los procesos y tendencias electorales, así como los problemas de la participación y representación política en torno del funcionamiento de los sistemas políticos de los Estados latinoamericanos. La elección del tema "América Latina: procesos y tendencias electorales" analizado en el "Dossier" fue motivada por el hecho de que en la región terminó un ciclo electoral, lo cual puso en evidencia nuevas tendencias que merecían ser analizadas (Krzywicka, 2015, pp. 13-16).

El principal tema abordado por los autores del tercer volumen (3/2016), elaborado bajo la coordinación científica de Renata Siuda-Ambroziak del Centro de Estudios Latinoamericanos de la Universidad de Varsovia, son las relaciones entre el Estado, la política y la religión en los países de América Latina. Lo que nos inspiró a dedicar un volumen a este tema fue un debate interdisciplinar que tuvo lugar en Lublin, Polonia, en los días 3 y 4 de noviembre de 2015 durante la II Conferencia Internacional Latinoamericanista dedicada a los determinantes y nuevos paradigmas de las relaciones entre política y religión en América Latina. Las coordinadoras del volumen invitaron a varios investigadores que se especializan en esta problemática para preparar artículos publicados en el "Dossier" titulado "América Latina: política y religión" (Krzywicka y Siuda-Ambroziak, 2016, pp. 13-17).

En el cuarto volumen (4/2017) de la revista Anuario Latinoamericano dominaron los temas relacionados con el problema de la violencia en México y Centroamérica. Los coordinadores científicos del “Dossier”, Pablo Marcos Moloeznik de la Universidad de Guadalajara en México y Paweł Trefler de la Escuela Superior Estatal de Europa Oriental de Przemyśl en Polonia, son especialistas en el campo de la seguridad y las fuerzas armadas en América Latina (Moloeznik y Trefler, 2017, pp. 13-19).

El volumen quinto $(5 / 2017)$ de nuestra revista es el resultado de investigaciones de los científicos quienes se dedican al análisis de la historia y el desarrollo de la disciplina de ciencia política. Sin duda, este tema es importante y visible en el debate actual entre los politólogos en América Latina y en Europa. Los artículos que conforman el "Dossier" titulado "América Latina: desarrollo y balance de la ciencia política" y coordinado por Pablo Bulcourf de la Universidad de Buenos Aires y Paulo Ravecca de la Universidad de la República en Montevideo forman una parte valiosa del debate actual, ciertamente enriqueciéndolo y complementándolo (Bulcourf, Krzywicka y Ravecca, 2017, pp. 17-31).

El sexto volumen del Anuario Latinoamericano, cuyo coordinador invitado fue Karol Derwich del Departamento de Estudios Latinoamericanos de la Universidad Jaguelónica, fue publicado en 2018. Los autores de los artículos del “Dossier” titulado „América Latina y el Caribe: fronteras y zonas fronteri-
Anuario Latinoamericano Ciencias Políticas y Relaciones Internacionales vol. 10,2020

pp. $229-238$

DOI: 10.17951/al.2020.10.229-238 
zas" se enfocaron en las cuestiones relacionadas con varios aspectos del funcionamiento, la dinámica y las amenazas de las fronteras y áreas fronterizas. Este tema es muy actual en el debate político y tiene particular importancia para los Estados de América Latina que enfrentan retos relacionados con las migraciones de personas, la economía ilícita o el crimen organizado (Derwich y Krzywicka, 2018, pp. 13-17).

El año 2019 fue muy especial para Anuario Latinoamericano - Ciencias Políticas y Relaciones Internacionales, ya que hemos entrado en el segundo lustro de la publicación de la revista y hemos publicado dos números voluminosos. El séptimo (7/2019), titulado "América Latina: continuidad y cambio en el escenario regional" y el cual coordiné personalmente, incluye en el "Dossier" los artículos cuyos autores realizaron un análisis multidimensional de los condicionamientos, el desarrollo y la dinámica del escenario político, económico, social y cultural actual de América Latina (Krzywicka, 2019, pp. 13-16). A su vez, el volumen octavo (8/2019) de Anuario Latinoamericano, dedicado al tema "América Latina: género y política", ha creado un espacio para el análisis científico, reflexión e intercambio de experiencias sobre el problema de género, un problema muy actual y relevante en la realidad de los países de América Latina, posibilitando además una multidimensional percepción de este tema desde la perspectiva de las ciencias sociales, con la idea de inspirar la discusión orientada al problema de equidad de género en el funcionamiento del Estado democrático y de la sociedad abierta. Los autores del volumen elaborado bajo la coordinación científica de María Elena Martin de la Universidad del Salvador en Buenos Aires y de la mía analizan, entre otros, problemas de la igualdad y diversidad en la teoría y práctica política, como también de la representación y participación política de las mujeres (Krzywicka y Martin, 2019, pp. 13-17).

En 2020, bajo las condiciones muy particulares de la pandemia del COVID-19, hemos elaborado dos consecutivos volúmenes de la revista Anuario Latinoamericano. El contenido del "Dossier" del noveno volumen (9/2020), titulado "América Latina: Venezuela" y preparado bajo mi coordinación científica, es el resultado de investigaciones de los científicos quienes se dedican al análisis de la historia, economía, política y relaciones internacionales de Venezuela. Sin duda, el Estado venezolano se muestra como un caso inspirador para un análisis multidimensional, además constantemente presente en el debate científico actual tanto en América Latina como en otras partes del mundo. En la sección "Dossier" nuestros lectores encontrarán artículos sobre las causas, manifestaciones, naturaleza y consecuencias de la crisis del Estado en Venezuela (Krzywicka, 2020, pp. 9-10).

El tema principal analizado en el "Dossier" del décimo volumen del Anuario Latinoamericano fue elaborado bajo la coordinación científica de Daniel Morales Ruvalcaba del Centro de Estudios Latinoamericanos de la Universidad Sun Yat-sen (China) y la mía. Los autores del volumen (10/2020) exploran el origen, la naturaleza, los objetivos y resultados de la iniciativa china de la Franja y la Ruta en América Latina (Krzywicka, 2020a, pp. 9-12). 
Cabe señalar que desde 2014, año tras año, el interés de los estudiosos por la publicación de los resultados de su investigación científica en las páginas de la revista Anuario Latinoamericano está aumentando, lo cual es el resultado de un trabajo constante y sistemático con el fin de elevar no solamente la calidad, pero también la visibilidad y el impacto de la revista.

Los volúmenes de Anuario Latinoamericano fueron presentados por los miembros del Equipo Editorial durante varios eventos científicos internacionales importantes para el campo de las investigaciones dedicadas a América Latina y el Caribe. La revista fue presentada durante los siguientes eventos: el VIII Congreso Internacional del Consejo Europeo de Investigaciones Sociales en América Latina (CEISAL) en 2016; el Simposio Internacional del Centro de Estudios Ibero-Americanos en la Universidad Carolina en Praga, 2016 (Ochab, 2017, pp. 289-292); el Seminario Latinoamericano: Estudios Latinoamericanos en Europa Central y Oriental en la Universidad Jaguelónica en Cracovia, en 2017 (Derwich, 2017, pp. 297-298); la Jornada de México en la Universidad Maria Curie-Skłodowska en Lublin, en 2017 (Krzywicka, 2017a, pp. 337-339); el XVIII Congreso Internacional de la Federación Internacional de Estudios sobre América Latina y el Caribe (FIEALC) en Belgrado, en 2017 (Ochab, 2017a, pp. 341-344); la I Jornada sobre la Historia y Desarrollo de la Ciencia Política en Iberoamérica en la Universidad de Granada, en 2018; el IV Congreso Internacional sobre Iberoamérica en Atenas, en 2018; los Simposios Internacionales "Encuentros Científicos con América Latina en la Facultad de Ciencias Políticas y Periodismo de la Universidad Maria Curie-Skłodowska en Lublin (2015, 2017, 2018 y 2019); el II Encuentro Internacional de Ciencia Política bajo el lema "Construyendo una disciplina desde la región: Insuficiencias en las Democracias Latinoamericanas ¿Cuáles son las alternativas?” en la Universidad del Cauca, en Colombia (2018); el 56 Congreso Internacional de Americanistas en 2018 en Salamanca; la III Conferencia Internacional Latinoamericanista en Lublin, organizada del 21 al 22 de noviembre de 2018 con motivo del quinto aniversario de la revista Anuario Latinoamericano - Ciencias Políticas y Relaciones Internacionales y del vigésimo quinto aniversario de la Facultad de Ciencias Políticas de la Universidad Maria Curie-Skłodowska; el II Congreso Latinoamericano Ignacio Domeyko en la Facultad de Estudios Internacionales y de Ciencias Políticas de la Universidad de Łódź, 15-16 de diciembre de 2018 en Polonia (Kosmynka y Stelmach, 2019, pp. 361-363).

En la primera mitad de 2019, tuve la oportunidad de presentar el Anuario Latinoamericano durante una visita científica en la Universidad de Guadalajara en México. La presentación de la revista se celebró en la biblioteca principal de la universidad, en la librería Carlos Fuentes. El evento fue organizado en cooperación con el Departamento de Estudios Políticos y el Departamento de Estudios Internacionales de la Universidad de Guadalajara (Krzywicka, 2019b, pp. 365-366). La revista también fue presentada durante la V Conferencia Cracoviana Latinoamericanista, la cual tuvo lugar en el Instituto de Estudios Americanos y la Diáspora Polaca de la Universidad Jaguelónica en Cracovia (Sawicka, 2019,
Anuario Latinoamericano Ciencias Políticas y Relaciones Internacionales vol. 10, 2020

pp. $229-238$

DOI: 10.17951/al.2020.10.229-238 
pp. 367-370), y durante el XIX Congreso de la Federación Internacional de Estudios sobre América Latina y el Caribe (FIEALC), organizado por la Universidad de Szeged en Hungría. La quinta y la sexta edición del Simposio Internacional "Encuentros Científicos con América Latina", que tuvieron lugar en la Facultad de Ciencias Políticas de la Universidad Maria Curie-Skłodowska de Lublin en mayo y octubre de 2019, también han brindado la oportunidad de promover la revista.

En la segunda mitad del 2019, tuve la oportunidad de presentar el Anuario Latinoamericano durante el IV Foro Internacional "Rusia e Iberoamérica en el mundo globalizante: historia y perspectivas", organizado los días 1-3 de octubre por el Centro de Estudios Iberoamericanos de la Universidad Estatal de San Petersburgo. Otro evento en el que tuve el privilegio de presentar la revista fue el Congreso Internacional "Innovación y desarrollo en el área de la Gran Bahía: oportunidades para América Latina” celebrado en la Universidad Sun Yat-sen, una de las diez mejores universidades chinas. El evento fue organizado por el Centro de Estudios de América Latina de la Escuela de Estudios Internacionales los días 15-16 de noviembre de 2019 en la ciudad de Zhuhai en la República Popular China (Krzywicka, 2019d, pp. 345-347).

Aparte del trabajo editorial, una forma de la actividad científica muy característica y singular que realiza el Equipo Editorial de la revista Anuario Latinoamericano - Ciencias Políticas y Relaciones Internacionales es la organización y el patrocinio de los eventos científicos que reúnen a investigadores, académicos y estudiantes interesados en la región de América Latina y cuyo objetivo es profundizar el conocimiento sobre América Latina y el Caribe. Entre las actividades más emblemáticas se encuentran los simposios internacionales organizados cíclicamente en la Facultad de Ciencias Políticas y Periodismo de la Universidad Maria Curie-Skłodowska en Lublin desde el año 2015 bajo el lema "Encuentros Científicos con América Latina”. Se han celebrado seis ediciones del evento. La primera tuvo lugar en marzo y abril de 2015. Los ponentes invitados fueron politólogos de tres países: México, Alemania y Suiza. En febrero de 2017 tuvo lugar la segunda edición del evento. Los ponentes invitados, los colaboradores de la revista, vinieron de Argentina y de la República Checa (Krzywicka, 2017, pp. 293-295). La tercera edición de los "Encuentros Científicos con América Latina" se ha celebrado en la ocasión del quinto aniversario de la revista Anuario Latinoamericano - Ciencias Políticas y Relaciones Internacionales en junio de 2018 en la Facultad de Ciencias Políticas de la UMCS (Krzywicka, 2018a, pp. 299-302). Las conferencias fueron impartidas por científicos de las universidades argentinas y polacas. Una parte integral del evento fue la exposición fotográfica titulada "América Latina y el Caribe: sociedad - cultura - naturaleza”. La exposición tuvo lugar del 6 de junio al 7 de julio de 2018. La quinta edición del Simposio Internacional los "Encuentros Científicos con América Latina" tuvo lugar los días 22 y 23 de mayo de 2019. En el evento asistieron los politólogos de los centros de investigación extranjeros de Israel y China, como también del Instituto de Estudios Americanos y la Diáspora Polaca de la Universidad Jaguelónica en Polonia 
(Krzywicka, 2019c, pp. 371-372). La última y sexta edición del simposio se celebró los días 22-25 de octubre de 2019. Durante los cuatro días del evento los participantes discutieron sobre las políticas de seguridad de los Estados de América Latina (Ochab, 2019, pp. 341-343). El evento contó con la participación de conferencistas, científicos e investigadores de los centros académicos extranjeros del Reino Unido y Lituania, así como de las universidades polacas de Łódź, Przemyśl, Cracovia y Lublin. Planeada para el año 2020 la séptima edición de los "Encuentros Científicos con América Latina” fue cancelada por causa de la pandemia del COVID-19.

Por mi iniciativa, como editora en jefe de la revista Anuario Latinoamericano - Ciencias Políticas y Relaciones Internacionales, se organizan en Lublin de manera cíclica las Conferencias Internacionales Latinoamericanistas. El más reciente de estos eventos se celebró en noviembre de 2018 sobre el tema "América Latina - la región de relaciones internacionales en el siglo XXI". Esta fue la tercera de las conferencias científicas organizadas con el objetivo de crear un foro del debate interdisciplinario e internacional para investigadores latinoamericanistas, así como el desarrollo y la promoción de la investigación en el área de ciencias políticas llevada a cabo por los científicos polacos sobre el tema de la región de América Latina y el Caribe (Krzywicka, 2019, pp. 357-359). A la conferencia asistieron politólogos de Argentina, Brasil, Colombia, México, la República Checa, España, Lituania, Alemania, Gran Bretaña y Polonia. Las Conferencias Internacionales Latinoamericanistas se inscriben en las actividades de la Facultad de Ciencias Políticas y Periodismo de la Universidad Maria Curie-Skłodowska realizadas con el fin de la internacionalización de la ciencia.

Los principios de aumentar la visibilidad y desarrollar el impacto de Anuario Latinoamericano en el entorno académico se realizan también a través de la presencia de la revista en varias bases internacionales de datos de referencia. Anuario Latinoamericano está indexado en las siguientes bases de datos: Latindex - Sistema Regional de Información en Línea para Revistas Científicas de América Latina, el Caribe, España y Portugal (desde 2015); Index Copernicus - International Journals Master List (desde 2015); ERIH PLUS - The European Reference Index for the Humanities and the Social Sciences (desde 2016); ROAD - Directory of Open Access Scholary Resources (desde 2016); CEJSH - Central European Journal of Social Sciences and Humanities (desde 2017); REDIB - Red Iberoamericana de Innovación y Conocimiento Científico (desde 2017); DOAJ - Directory of Open Access Journals (desde 2019); Crossref y en la Bibliografía Científica Polaca, PBN-POL-index. La información sobre la revista está disponible en las páginas web de la Asociación de Ciencia Política Internacional (IPSA) y la Asociación Latinoamericana de Ciencia Política (ALACIP). Nuestra revista ha sido incluida también, como uno de los pocos periódicos latinoamericanistas del área de las ciencias políticas en Europa, en el informe titulado "Revistas europeas de Estudios Latinoamericanos - Informe preparado para el $8^{\circ}$ Congreso del CEISAL, Salamanca 2016" de la Red Europea de Información y Documentación sobre América Latina (REDIAL).
Anuario Latinoamericano Ciencias Políticas y Relaciones Internacionales vol. 10, 2020

pp. $229-238$

DOI: 10.17951/al.2020.10.229-238 
Muy significativos para el aumento de la calidad, visibilidad e impacto de la revista Anuario Latinoamericano y para garantizar la continuidad de su publicación fueron las subvenciones ministeriales para los años 2019-2020, conseguidos en dos concursos organizados por el Ministerio de Ciencia y Educación Superior de Polonia en 2018. La revista recibió una alta calificación de 95 puntos en la escala de 100 puntos, lo que permitió aprovechar fondos del programa "Apoyo a las revistas científicas". Además, como una forma de aprecio por cinco años de la ardua labor científica y editorial, Anuario Latinoamericano fue introducido en la lista de revistas científicas del Ministerio de Ciencia y Educación Superior. Esto es una muestra del impacto de nuestra revista en la divulgación de la ciencia en Polonia, así como también en la internacionalización de los estudios latinoamericanos.

Aprovechando el privilegio de ser la fundadora y editora en jefe de la revista Anuario Latinoamericano - Ciencias Políticas y Relaciones Internacionales, quiero expresar mi profunda gratitud por la fructífera y profesional colaboración en el lanzamiento de los diez volúmenes de nuestra revista a todos autores y evaluadores de los artículos, a los coordinadores científicos de las secciones "Dossier" y a los miembros del Comité Científico. Nuestra cooperación científica constituye una importante contribución al desarrollo de los estudios internacionales sobre la región de América Latina y el Caribe. Queda por esperar que las páginas de nuestra revista sigan siendo el lugar de importantes y actuales debates científicos para apoyar la colaboración académica y mayor internacionalización de la investigación científica, contribuyendo así al desarrollo de la disciplina de las ciencias políticas.

Pensando en el trabajo realizado hasta ahora y sobre el futuro de la revista quiero dar las gracias a los miembros del Equipo Editorial por su esfuerzo y determinación para llevar a cabo el trabajo editorial.

El desarrollo de la revista Anuario Latinoamericano no sería posible sin el apoyo constante de las autoridades de la Universidad Maria Curie-Skłodowska de Lublin (UMCS) y de la Facultad de Ciencias Políticas y Periodismo de la UMCS, ni tampoco sin efectiva cooperación con la Editorial de la UMCS.

Por último, quiero dar las gracias a todos lectores de la revista Anuario Latinoamericano - Ciencias Políticas y Relaciones Internacionales, porque nuestros esfuerzos solo se verán coronados por el éxito si su resultado es de utilidad para nuestros lectores.

\section{Referencias}

Ardila, M. y Krzywicka, K. (2014). América Latina: cambios a nivel regional y en su inserción internacional. Presentación. Anuario Latinoamericano - Ciencias Políticas $y$ Relaciones Internacionales, vol. 1, pp. 13-15. DOI:10.17951/al.2014.1.13

Bulcourf, P., Krzywicka, K. y Ravecca, P. (2017). Reconstruyendo la Ciencia Política en América Latina. Presentación. Anuario Latinoamericano - Ciencias Políticas y Relaciones Internacionales, vol. 5, pp. 17-31. DOI: 10.17951/al.2017.5.17 
Derwich, K. (2017). Informe del Seminario Latinoamericano en el Departamento de Estudios Latinoamericanos de la Universidad Jaguelónica en Cracovia, 3 de abril de 2017. Anuario Latinoamericano - Ciencias Políticas y Relaciones Internacionales, vol. 4, pp. 297298. DOI: 10.17951/al.2017.4.297

Derwich, K. y Krzywicka, K. (2018). Fronteras y zonas fronterizas en América Latina y el Caribe. Presentación. Anuario Latinoamericano - Ciencias Políticas y Relaciones Internacionales, vol. 6, pp. 13-17. DOI: 10.17951/al.2018.6.13-17

Kosmynka, S. y Stelmach, M. (2019). El II Congreso Latinoamericano Ignacio Domeyko en la Facultad de Estudios Internacionales y de Ciencias Políticas de la Universidad de Łódź, 15-16 de diciembre de 2018, Łódź, Polonia. Anuario Latinoamericano - Ciencias Políticas $y$ Relaciones Internacionales, vol. 7, pp. 361-363. DOI: 10.17951/al.2019.7.361-363

Krzywicka, K. (2015). América Latina: procesos y tendencias electorales. Presentación. Anuario Latinoamericano - Ciencias Políticas y Relaciones Internacionales, vol. 2, pp. 13-16. DOI: $10.17951 /$ al.2015.2.13

Krzywicka, K. y Siuda-Ambroziak, R. (2016). América Latina: política y religión. Presentación. Anuario Latinoamericano - Ciencias Políticas y Relaciones Internacionales, vol. 3, pp. 13-17. DOI: $10.17951 /$ al.2016.3.13

Krzywicka, K. (2017). Informe de la segunda edición de Los Encuentros Científicos con América Latina en la Facultad de Ciencias Políticas de la Universidad Maria Curie-Skłodowska en Lublin, Polonia, 31 de enero - 3 de febrero de 2017. Anuario Latinoamericano - Ciencias Políticas $y$ Relaciones Internacionales, vol. 4, pp. 293-295. DOI: 10.17951/al.2017.4.293

Krzywicka, K. (2017a). Informe de la Jornada de México en la Facultad de Ciencias Políticas de la Universidad Maria Curie-Skłodowska en Lublin, Polonia, 7 de junio de 2017. Anuario Latinoamericano - Ciencias Politicas y Relaciones Internacionales, vol. 5, pp. 337-339. DOI: $10.17951 /$ al.2017.5.337

Krzywicka, K. (2018). Anuario Latinoamericano - Ciencias Políticas y Relaciones Internacionales. Jakość, widoczność, oddziaływanie. Ameryka Łacińska, 2 (100), pp. 158-167.

Krzywicka, K. (2018a). El quinto aniversario de la revista científica "Anuario Latinoamericano - Ciencias Políticas y Relaciones Internacionales". Anuario Latinoamericano - Ciencias Politicas y Relaciones Internacionales, vol. 6, pp. 299-302. DOI: 10.17951/ al.2018.6.299-302

Krzywicka, K. (2019). Continuidad y cambio en el escenario regional de América Latina y el Caribe. Presentación. Anuario Latinoamericano - Ciencias Políticas y Relaciones Internacionales, vol. 7, pp. 13-16. DOI: 10.17951/al.2019.7.13-16

Krzywicka, K. (2019a). La III Conferencia Internacional Latinoamericanista en la Facultad de Ciencias Políticas de la Universidad Maria Curie-Skłodowska, 21-22 de noviembre de 2018, Lublin, Polonia. Anuario Latinoamericano - Ciencias Políticas y Relaciones Internacionales, vol. 7, pp. 357-359. DOI: 10.17951/al.2019.7.357-359

Krzywicka, K. (2019b). El II Congreso Internacional de Derechos Humanos, Justicia y Migración, 11-16 de febrero de 2019, Guadalajara, México. Anuario Latinoamericano - Ciencias Políticas y Relaciones Internacionales, vol. 7, pp. 365-366. DOI: 10.17951/ al.2019.7.365-366

Krzywicka, K. (2019c). La V edición del Simposio Internacional "Encuentros Científicos con América Latina" en la Facultad de Ciencias Políticas de la Universidad Maria Curie-Skłodowska, 22-23 de mayo 2019, Lublin, Polonia. Anuario Latinoamericano Ciencias Políticas y Relaciones Internacionales, vol. 7, 2019, pp. 371-372. DOI: 10.17951/ al.2019.7.371-372
Anuario Latinoamericano Ciencias Políticas y Relaciones Internacionales vol. 10,2020

pp. $229-238$

DOI: 10.17951/al.2020.10.229-238 
Krzywicka, K. (2019d). Congreso Internacional "Innovación y desarrollo en China: oportunidades para América Latina", Centro de Estudios Latinoamericanos, Escuela de Estudios Internacionales, Universidad Sun Yat-sen, 15-16 de noviembre de 2019, Zhuhai, República Popular China. Anuario Latinoamericano - Ciencias Políticas y Relaciones Internacionales, vol. 8, pp. 345-347. DOI: 10.17951/al.2019.8.345-347

Krzywicka, K. (2020). Editorial. Anuario Latinoamericano - Ciencias Políticas y Relaciones Internacionales, vol. 9, pp. 9-10. DOI: 10.17951/al.2020.9.9-10

Krzywicka, K. (2020a). Editorial. Anuario Latinoamericano - Ciencias Políticas y Relaciones Internacionales, vol. 10, pp. 9-12. DOI: 10.17951/al.2020.10.9-12

Krzywicka, K. y Martín, M. E. (2019). América Latina: género y política. Presentación. Anuario Latinoamericano - Ciencias Políticas y Relaciones Internacionales, vol. 8, pp. 13-17. DOI: 10.17951/al.2019.8.13-17

Moloeznik, M. P. y Trefler, P. (2017). América Latina: violencia en México y Centroamérica. Presentación. Anuario Latinoamericano - Ciencias Políticas y Relaciones Internacionales, vol. 4, pp. 13-19. DOI: 10.17951/al.2017.4.13

Ochab, M. (2017). Informe del Simposio Internacional Las relaciones entre Europa Central y América Latina. Contextos históricos, Centro de Estudios Ibero-Americanos de la Universidad Carolina, Praga, 15-16 septiembre de 2016. Anuario Latinoamericano - Ciencias Políticas y Relaciones Internacionales, vol. 4, pp. 289-292. DOI: 10.17951/ al.2017.4.289

Ochab, M. (2017a). Informe del XVIII Congreso de la Federación Internacional de Estudios sobre América Latina y el Caribe (FIEALC) en Belgrado, Serbia, 25-28 de julio de 2017. Anuario Latinoamericano - Ciencias Políticas y Relaciones Internacionales, vol. 5, pp. 341344. DOI: 10.17951/al.2017.5.341

Ochab, M. (2019). La VI edición del Simposio Internacional "Encuentros Científicos con América Latina" en la Facultad de Ciencias Políticas y Periodismo de la Universidad Maria Curie-Skłodowska, 22-25 de octubre 2019, Lublin, Polonia. Anuario Latinoamericano - Ciencias Políticas y Relaciones Internacionales, vol. 8, pp. 341-343. DOI: 10.17951/ al.2019.8.341-343

Sawicka, M. (2019). The 5th Cracovian Conference of Latin Americanists Violence in Latin America. Challenges and Perspectives for the Future, 5-6th of April 2019, Cracow, Poland. Anuario Latinoamericano - Ciencias Políticas y Relaciones Internacionales, vol. 7, pp. 367370. DOI: 10.17951/al.2019.7.367-370

Katarzyna Krzywicka Facultad de Ciencias Políticas y Periodismo Universidad Maria Curie-Skłodowska Lublin, Polonia katarzyna.krzywicka@umcs.edu.pl https://orcid.org/0000-0001-5623-5384 